\title{
Funcionalidadee Incapacidade de Mulheres Submetidasà Cirurgia Oncológica Mamária: Uso da Classificação Internacional de Funcionalidade, Incapacidade e Saúde
}

https://doi.org/10.32635/2176-9745.RBC.2021v67n4.1488

\author{
Functionality and Disability of Women Undergoing Breast Cancer Surgery: Utilization of the International Classification of \\ Functionality, Disability, and Health \\ Funcionalidad y Discapacidad de las Mujeres Sometidas a Cirugía de Cáncer de Mama: Uso de la Clasificación Internacional \\ del Funcionamiento, la Discapacidad y la Salud
}

Lucas dos Santos Galaverna'; Lilian de Fátima Dornellas²; Maria Selma Duarte Nogueira ${ }^{3}$; Rogério Mendonça de Carvalho4; Frederico Tadeu Deloroso5; Wallisen Tadashi Hattori ${ }^{6}$; Eliane Maria de Carvalho ${ }^{7}$

\section{RESUMO}

Introduçáo: O uso da Classificação Internacional de Funcionalidade, Incapacidade e Saúde (CIF) orienta o raciocínio clínico e o planejamento do cuidado oncológico. Objetivo: Determinar a funcionalidade e a incapacidade de mulheres mastectomizadas, por meio da aplicaçáo de questionários de qualidade de vida e autoestima, perimetria e biofotometria, convertidos pelo método de ligaçáo com as categorias da CIF. Método: Estudo transversal com 30 mulheres mastectomizadas com idade acima de 40 anos, independentemente do tempo pós-operatório, avaliadas por questionários de qualidade de vida, disfunção de membro superior, escala de autoestima de Rosenberg, biofotometria e perimetria, convertidos pelo método de ligação com as categorias da CIF. Resultados: As mulheres submetidas à cirurgia oncológica mamária apresentaram frequentemente alteraçôes na estrutura do corpo (pelos (86,6\%), mama e mamilos $(76,6 \%)$ ) e na função do corpo (dor no membro superior (83,3\%) e redução da mobilidade do ombro nos movimentos de abdução (100\%) e flexão (93\%)); limitaçóes nas atividades (concluir rotina diária (73,3\%), transportar $(76,6 \%)$, rodar ou torcer utilizando as mãos $(80 \%)$ ), lavar partes do corpo $(73,3 \%)$, realizar tarefas domésticas $(73,3 \%)$ ); restriçốes na participação (lidar com responsabilidades $(73,3 \%)$ ); e presença de facilitadores ambientais (relação familiar e serviços de saúde). Conclusão: Mulheres submetidas à cirurgia oncológica mamária apresentam incapacidades relacionadas a alteraçóes de estrutura e funçáo do corpo, limitaçóes de atividades, restrição na participação e presença de facilitadores ambientais, sugerindo impactos além da condição de saúde na vida delas.

Palavras-chave: Classificação Internacional de Funcionalidade, Incapacidade e Saúde; Sobreviventes de Câncer; Neoplasias da Mama; Modalidades de Fisioterapia.

\section{ABSTRACT}

Introduction: The utilization of the International Classification of Functioning, Disability, and Health (ICF) guides the clinical reasoning and planning of cancer care. Objective: To determine the functionality and disability of women who underwent mastectomy, through the application of quality of life and self-esteem, perimetry, and biophotometry questionnaires, converted by the linking rules to the ICF categories. Method: Cross-sectional study with 30 women who underwent mastectomy, over 40 years old regardless of postoperative time, assessed through quality-of-life questionnaires, upper limb dysfunction, Rosenberg's self-esteem scale, biophotometry, and perimetry converted to the ICF conceptual model by the linking rules. Results: Women who underwent breast cancer surgery frequently presented changes in their body structure (hair $(86.6 \%)$, breast and nipples $(76.6 \%)$ ), and in their body functions, (pain in upper limb $(83.3 \%)$ and reduced shoulder mobility in abduction (100\%) and flexion (93\%) movements)); limitations in their daily activities (completing the daily routine $(73.3 \%)$, transport $(76.6 \%)$, turn or twist the hands (80\%), washing body parts (73.3\%), doing housework (73.3\%)); limited participation (handling responsibilities (73.3\%)), and environmental facilitators (family relationships and access to health services). Conclusion: Women who underwent breast cancer surgery had disabilities related to their body structure and functions, limitation of activities and participation and presence of environmental facilitators, suggesting impacts in the lives of these women beyond the health condition.

Key words: International Classification of Functioning, Disability and Health; Cancer Survivors; Breast Neoplasms; Physical Therapy Modalities.

\section{RESUMEN}

Introducción: El uso de la Clasificación Internacional del Funcionamiento, la Discapacidad y la Salud (CIF) orienta el razonamiento clínico y la planificación del tratamiento del cáncer. Objetivo: Determinar el funcionamiento y la discapacidad de las mujeres mastectomizadas, mediante la aplicación de cuestionarios de calidad de vida y autoestima, perimetría y biofotometría convertidos por el método de conexión con las categorías de la CIF. Método: Estudio transversal con 30 mujeres mastectomizadas, mayores de 40 ańos (independientemente del tiempo postoperatorio), evaluadas a través de cuestionarios de calidad de vida, disfunción del miembro superior, escala de autoestima de Rosenberg, biofotogrametría y perimetría convertida al modelo conceptual CIF por el método de conexión. Resultados: Las mujeres sometidas a cirugía de cáncer de mama presentaron alteraciones frecuentes en la estructura del cuerpo (cabello (86,6\%), dolor en las mama y pezones $(76,6 \%)$ ); en las funciones del cuerpo (dolor en el miembro superior $(83,3 \%)$ y movilidad reducida del hombro en movimientos de abducción $(100 \%)$ y flexión $(93 \%)$ ); limitaciones en sus actividades (llevar a cabo rutinas diarias $(73,3 \%)$, en el transporte $(76,6 \%)$, girar o torcer las manos o los brazos $(80 \%)$, lavar partes del cuerpo $(73,3 \%)$, realizar tareas domésticas $(73,3 \%)$ ); restricción de participación, (manejo de responsabilidades $(73,3 \%)$ ) y los facilitadores ambientales (relaciones familiares y el acceso a los servicios de salud). Conclusión: Las mujeres sometidas a cirugía de cáncer de mama presentan discapacidades relacionadas con cambios en la estructura y función corporal, limitaciones de actividad, restricciones de participación y la presencia de facilitadores ambientales que sugieren impactos más allá de la condición de salud de estas mujeres.

Palabras clave: Clasificación Internacional del Funcionamiento, de la Discapacidad y de la Salud; Supervivientes de Cáncer; Neoplasias de la Mama; Modalidades de Fisioterapia.

\footnotetext{
1,3,4,5,6,7Universidade Federal de Uberlândia. Uberlândia (MG), Brasil. E-mails: lucas_galaverna98@hotmail.com; sel.duart@gmail.com; rocarvalho@ufu.br; fredericodeloroso@ufu.br; wallhattori@gmail.com; elianemc@ufu.br. Orcid iD: https://orcid.org/0000-0001-5581-7876; Orcid iD: https://orcid.org/0000-0002-32360815; Orcid iD: https://orcid.org/0000-0002-0326-1236; Orcid iD: https://orcid.org/0000-0001-8714-2156; Orcid iD: https://orcid.org/0000-0002-6904-0292; Orcid iD: https://orcid.org/0000-0002-3977-9117

2Universidade Federal do Mato Grosso do Sul. Campo Grande (MS), Brasil. E-mail: liliandefatima@hotmail.com. Orcid iD: https://orcid.org/0000-0001-8662-5463 Endereço para correspondência: Lucas dos Santos Galaverna. Rua Pedro Silotto, 108 - Centro. Serra Negra (SP), Brasil. CEP 13930-000. E-mail: lucas_galaverna98@hotmail.com
} 


\section{INTRODUÇÃO}

As incapacidades de mulheres sobreviventes ao câncer de mama envolvem prejuízos biopsicossociais e uma ferramenta de referência mundial que pode orientar tanto no processo avaliativo quanto na intervenção e no acompanhamento fisioterapêutico é a Classificaçáo Internacional de Funcionalidade, Incapacidade e Saúde $(\mathrm{CIF})^{1}$.

A CIF aborda componentes de saúde que permitem a visualização do indivíduo como um todo, além de ajudar na construção de projetos terapêuticos individualizados e orientados às necessidades dos usuários, na estruturação e no fortalecimento da inserção de indicadores de saúde em Sistemas de Informação em Saúde ${ }^{2,3}$. No estudo de Lourenço et al. ${ }^{4}$, foi verificado, por meio do questionário Whodas 2.0, que $94 \%$ das mulheres sobreviventes ao câncer de mama apresentaram algum tipo de deficiência associada à maior intensidade de fadiga e à pior qualidade do sono e de vida. Esses problemas identificados demonstram a relevância e a necessidade de integrar informaçóes, além da condição de saúde relacionada à funcionalidade nas avaliaçóes clínicas e condutas terapêuticas.

Embora seja importante essa abordagem, estudos acerca da utilização e disseminaçáo da CIF demonstram sua pouca inserção na prática clínica nessa população, ou mensuram apenas um componente ${ }^{5,6}$. Castro et al. ${ }^{7}$, com o objetivo de avaliar a incapacidade e funcionalidade de mulheres em tratamento de câncer de mama, baseando-se em questionários advindos da CIF, como core set para câncer de mama e o Whodas 2.0, relataram que a utilizaçáo desses instrumentos possui pontos positivos, mas também há pontos negativos, como a falta de praticidade e conhecimento de seu uso. Na revisão publicada por Santos et al. ${ }^{5}$, foi observado que a utilização da CIF em mulheres com câncer de mama é baixa, sendo justificada pelo desconhecimento profissional, impressão de alta complexidade, desinteresse, baixo envolvimento de gestão e baixa iniciativa macroinstitucional, entretanto, todos os estudos destacaram a importância da visão biopsicossocial na abordagem terapêutica.

Considerando os desafios quanto ao uso da CIF, este estudo tem como pergunta norteadora: a ficha de avaliação fisioterapêutica de um serviço público no interior de Minas Gerais, que possui instrumentos comuns na prática clínica em mulheres com câncer de mama, atinge todos os componentes da CIF? Dessa forma, o objetivo do estudo foi determinar a funcionalidade e a incapacidade de mulheres submetidas à cirurgia oncológica mamária, por meio da aplicação de uma ficha de avaliação fisioterapêutica já estruturada com questionários de qualidade de vida, questionário de disfunção de ombro, cotovelo e mão, perimetria e biofotometria e a ligação com as categorias da CIF.

\section{MÉTODO}

Estudo transversal quantitativo, com amostra por conveniência, aprovado pelo Comitê de Ética em Pesquisa com Seres Humanos da Universidade Federal de Uberlândia, Minas Gerais, sob o número de parecer 2.731.732.

Trinta voluntárias diagnosticadas com câncer de mama submetidas à cirurgia oncológica mamária em hospitais públicos e privados da Região do Triângulo Mineiro e Alto Paranaíba, sem distinção de raça ou etnia, com idades superiores a 40 anos, em início de tratamento fisioterapêutico, independentemente do tempo pós-cirúrgico, participaram da presente pesquisa.

Os critérios de inclusão foram voluntárias submetidas à cirurgia oncológica mamária radical e conservadora e a assinatura do Termo de Consentimento Livre e Esclarecido. Já os critérios de exclusão foram presença de lesôes e/ou ferimentos na regiáo da mama, metástase do câncer primário e fraturas patológicas de membros superiores (MMSS).

A avaliação foi composta pelos seguintes instrumentos: 1. Questionário Disabilities of Arm, Shoulder and Hand (DASH): composto por 30 questôes autoaplicáveis, com itens que informam sobre o grau de dificuldade no desempenho de atividades; intensidade dos sintomas de dor, fraqueza, rigidez e parestesia; comprometimento de atividades sociais; dificuldade para dormir; e comprometimento psicológico, tendo como referência a semana anterior à aplicação do instrumento. Cada item do questionário possui cinco possibilidades de respostas, variando de 1 para nenhuma dificuldade ou sintoma até 5 para incapacidade ou sintoma extremo. O escore é obtido pela somatória dos valores atribuídos a cada item, diminuindo 30 e dividindo a subtração por 1,2. Escore alto indica grande disfunção ${ }^{8}$.

2. Questionários de Qualidade de Vida da European Organization for Research and Treatment of Cancer (EORTC) versóes QLQ-30 e QLQ-BR 23: compostos por um total de 53 questóes cuja abordagem inclui sintomas específicos do câncer; efeitos colaterais do tratamento; estado psicológico; funcionamento físico; interação pessoal; e saúde global. As questôes 1 a 28 e 31 a 53 são pontuadas de 1 a 4, sendo 1 - não; 2pouco; 3- moderadamente; e 4- muito. As questôes 29 e 30 são referentes à classificação de qualidade de vida e estado de saúde de 0 a 7 , sendo 0 - péssimo e 7- ótima. Por fim, são somados os valores atribuídos à cada questáo; quanto maior o valor, maior a qualidade de vida, sem categorização específica?. 
3. Escala de autoestima de Rosenberg (EAR): constituída por dez questóes de múltipla escolha referentes à autoimagem positiva e autodepreciação. Para ambos os questionários, se atribui um valor de resposta que mais representa o estado atual da voluntária. Cada assertiva do questionário possui quatro opçóes de resposta, classificadas em "concordo totalmente" até "discordo totalmente". Ao final da aplicação, são somados os valores atribuídos à cada assertiva, de modo a classificar a autoestima global, sendo alta com escore acima de 31 pontos, média entre 21 e 30 pontos, e baixa com escores inferiores a 20 pontos $^{10}$.

4. Biofotogrametria: garante a precisão nas mensuraçôes da amplitude de movimento (ADM) de extensão, flexão e abdução de ombro e flexão de cotovelo, sendo tais movimentos analisados pela fotografia de pontos anatômicos específicos e padronizados no software Kinovea versão 0.8.15 de 2014. O método possui alta confiabilidade e reprodutibilidade inter e intra-avaliadores. As fotos foram retiradas com câmera fotográfica de alta resolução da marca Canon modelo EOS Rebel T6 EF-S 18-55 f/3.5-5.6 III, focalizando os marcadores cilíndricos de alto relevo com $15 \mathrm{~mm}$ de diâmetro ${ }^{11}$.

5. Perimetria: realizada bilateralmente a cada 5 centímetros com início referenciado pela dobra do punho em 8 pontos demarcados ascendentemente.

Seguindo o modelo conceitual da CIF, os componentes "estrutura e função do corpo" foram contemplados por dados da cirurgia, questôes da EAR, questôes do questionário DASH, questóes dos questionários de qualidade de vida, biofotometria e perimetria. "Atividade e participação" foram contempladas nos questionários DASH, EAR e QLQ. Quanto aos "fatores ambientais", envolveram perguntas sobre serviços de saúde e relaçóes familiares e, por fim, os "fatores pessoais" colhidos por meio da identificação da paciente, incluindo hábitos de vida (etilismo, tabagismo).

As voluntárias do estudo compareceram ao ambulatório de Fisioterapia do Hospital de Clínicas - Hospital do Câncer da Universidade Federal de Uberlândia - em seguimento ao tratamento para câncer de mama. Antes de iniciarem a intervenção fisioterapêutica, foram convidadas a participar da pesquisa e, após o aceite e assinatura do Termo de Consentimento Livre e Esclarecido, foram avaliadas com os instrumentos supracitados.

Toda a coleta de dados foi realizada em um atendimento de 50 minutos. A equipe de avaliadores foi composta por quatro graduandos previamente treinados e padronizados. Primeiramente, foram coletadas informaçōes pessoais e da cirurgia (idade, atividade ocupacional, estado civil, escolaridade, renda familiar, queixa principal, ano de diagnóstico do câncer, histórico familiar, medicamentos em uso, hábitos de saúde, realização de tratamento coadjuvante, complicaçóes associadas, lado operado e de dominância, e aspecto da cicatriz). Posteriormente, foram aplicados os questionários DASH, EORTC QLQ-30, EORTC QLQ-BR-23 e EAR, em um ambiente isolado, garantindo a privacidade e a confidencialidade das respostas.

A perimetria e a biofotogrametria foram realizadas com a paciente em posiçáo sentada. A perimetria foi padronizada com a marcaçáo de 8 pontos a cada 5 centímetros de maneira ascendente com referência primária na dobra do punho e, após a marcação, foram medidas as circunferências em cada ponto. Já a biofotogrametria foi realizada em ambiente com boa iluminaçáo e distância entre câmera versus paciente pré-estabelecido, com pontos anatômicos demarcados por marcadores cilíndricos de PVC (acrômios, epicôndilos laterais e mediais do úmero, processos estiloides do rádio e ulna, e centro da dobra do punho) pré-definidos. As voluntárias foram fotografadas em posiçáo de mãos padronizadas em vista frontal para análise da abdução de ombro, e de perfil para verificação da flexão e extensáo de ombro e flexão de cotovelo. As fotos foram inseridas no software Kinovea ${ }^{\oplus}$ e, já com os pontos demarcados, eram selecionados o eixo do movimento e a mensuração da angulação a partir de uma reta traçada perpendicularmente ao solo e outra passando pelo ponto anatômico referenciado.

Todos os dados coletados foram tabulados e realizado o método de ligação entre cada assunto avaliado e seus respectivos componentes de saúde identificados na CIF. Todos os instrumentos, DASH, EAR, QLQ, perimetria, biofotogrametria e os dados da anamnese receberam categorias da CIF de acordo com os linking rules estabelecidos por Cieza et al. ${ }^{12}$, realizados por três pesquisadores independentes, os quais reuniram e elencaram aquelas categorias compatíveis por ao menos dois pesquisadores. Para os questionários QLQ, DASH e EAR, cada questão recebeu uma categoria, ao passo que a biofotometria e a perimetria receberam uma categoria para todo o instrumento.

Para cada categoria, foram estabelecidos qualificadores que melhor representassem a condição da paciente. Para os questionários de qualidade de vida, DASH e autoestima, os qualificadores seguiram a ordem de resposta dos questionários, pois cada questão possuía cinco opções de respostas com as mesmas perspectivas da CIF, considerando a ordem de menor para maior comprometimento. Já para a perimetria, consideraram-se a porcentagem de diferença de valor entre os membros no mesmo ponto e o grau de ADM mensurado na biofotometria, com base no modelo percentual recomendado pela CIF. Para o componente 
"atividade e participação", avaliou-se apenas o desempenho das pacientes, pois muitas categorias contempladas estavam presentes nos questionários autorrespondidos. Para a obtenção dos valores das análises estatísticas do componente "estrutura corporal", considerou-se apenas o primeiro qualificador, haja vista que este representa o grau de deficiência da mulher, contudo, os demais qualificadores caracterizaram a amostra.

Foi realizada uma análise descritiva sobre os qualificadores de cada categoria da CIF, por meio de percentuais de respostas. Para análise estatística, aplicou-se o teste qui-quadrado de aderência para avaliar a frequência das categorias das variáveis da funcionalidade, considerando aquelas que apresentaram porcentagem de algum nível de dificuldade, representadas pelos valores de média e mediana de cada categoria para as 30 voluntárias analisadas. Para todas as análises, adotou-se o nível de significância de 5\%. Utilizou-se o programa de análises SPSS versão 21.

\section{RESULTADOS}

Trinta voluntárias participaram do estudo com média de idade de 54,7 \pm 9,2 anos. A caracterizaçáo da amostra segue detalhada na Tabela 1 .

Tabela 1. Caracterização da amostra de 30 voluntárias* submetidas à cirurgia oncológica mamária

\begin{tabular}{lc}
\hline \multicolumn{1}{c}{ Dados } & $\mathbf{N}(\%)$ \\
\hline Idade & $2(6,6)$ \\
$38-40$ anos & $8(26,6)$ \\
$41-50$ anos & $12(40)$ \\
$51-60$ anos & $6(20)$ \\
$61-70$ anos & $2(6,6)$ \\
Acima de 70 anos & \\
\hline Estado civil & $6(20)$ \\
Solteira & $16(53,3)$ \\
Casada & $3(10)$ \\
Divorciada & $5(16,6)$ \\
Viúva & \\
\hline Tempo entre cirurgia e avaliação & $15(50)$ \\
$0-4$ meses & $5(16,6)$ \\
$5-9$ meses & $5(16,6)$ \\
$10-14$ meses & $1(3,3)$ \\
$15-19$ meses & $4(13,3)$ \\
Acima de 20 meses & \\
\hline Raça & $28(93,3)$ \\
Caucasiana & $2(6,7)$ \\
Negra &
\end{tabular}

(*) Ausência de vícios (etilismo e tabagismo).
Os instrumentos utilizados para a avaliação abordaram todos os componentes da CIF, sendo que "funçáo corporal" foi a que apresentou maior prevalência de alteraçôes em detrimento do maior número de categorias utilizadas, entre todas as elencadas pelo método de ligação (33; 46\%), seguida dos componentes "atividade e participação" (30; 41\%), "estrutura corporal” (7; 10\%) e "fatores ambientais" (2; 3\%).

Com relaçáo à "estrutura corporal”, todas as voluntárias apresentaram alguma alteração, de natureza relacionada principalmente à ausência parcial de mamas e mamilos (23; 76,6\%), ausência parcial de pelos (26;86,6\%), com a presença de mudança qualitativa, incluindo acúmulo de líquidos, principalmente nas regióes do ombro (30; $100 \%)$, cotovelo $(30 ; 100 \%)$ e punho $(30 ; 100 \%)$, em ambos os lados, mas com maior frequência no lado esquerdo (18; 60\%) (Tabela 2).

O componente "função corporal" demonstrou que grande parte das voluntárias apresentou alguma alteração, sendo as mais frequentemente acometidas: dor no peito $(21 ; 70 \%)$, dor no membro superior $(25 ; 83,3 \%)$, mobilidade do ombro principalmente nos movimentos de abdução $(30 ; 100 \%)$ e flexão (28; 93\%), força muscular de MMSS $(25 ; 83,3 \%)$ e nível de energia $(23 ; 76,7 \%)$ (Tabela 3).

Quanto à "atividade", as limitaçóes comumente relatadas pelas voluntárias foram concluir a rotina diária (22; 73,3\%), transportar (23; 76,6\%), alcançar $(21 ; 70 \%)$, rodar ou torcer utilizando as mãos $(24 ; 80 \%)$, lavar partes do corpo $(22 ; 73,3 \%)$ e realizar tarefas domésticas (22; 73,3\%). Já em "participação", a principal restrição foi lidar com responsabilidades $(73,3 \%)$ (Tabela 4). Em relação aos "fatores ambientais", o acesso aos serviços de saúde (e580) foi considerado como um facilitador completo, entre todas as voluntárias.

\section{DISCUSSÃO}

O presente estudo verificou que mulheres submetidas à cirurgia oncológica mamária apresentam incapacidades com prejuízos biopsicossociais.

As "estruturas corporais" mais acometidas das mulheres avaliadas, representadas por mama, mamilo (s6302) e edema (s420) apresentaram alteraçóes qualificadas como grave, e isso está relacionado, principalmente, à extirpação da mama, à magnitude da cirurgia e aos possíveis surgimentos de complicaçóes como o linfedema ${ }^{13}$. Entre as funçóes corporais mais frequentes e com maior gravidade, destacaram-se a mobilidade de uma única articulação (b7100), principalmente naqueles referentes aos movimentos de abdução e flexão do ombro, bem como a força de músculos isolados (b7300). Esse achado também 
Tabela 2. Frequência das categorias do domínio estrutura corporal da CIF, de acordo com os qualificadores de 30 voluntárias submetidas à cirurgia oncológica mamária

\begin{tabular}{|c|c|c|c|c|c|c|c|c|c|c|c|c|c|c|c|c|c|c|c|c|c|}
\hline \multicolumn{22}{|c|}{ s- Estrutura corporal } \\
\hline & & \multicolumn{8}{|c|}{$1^{\circ}$ Qualificador da CIF } & \multirow{2}{*}{\multicolumn{5}{|c|}{$\begin{array}{c}2^{\circ} \text { Qualificador da CIF } \\
\text { Natureza } \\
\text { da deficiência }\end{array}$}} & \multirow{2}{*}{\multicolumn{4}{|c|}{$\begin{array}{c}3^{\circ} \text { Qualificador } \\
\text { da CIF } \\
\text { Localização da } \\
\text { deficiência }\end{array}$}} & \multirow{3}{*}{ Média } & \multirow{3}{*}{ Mediana } & \multirow{3}{*}{ p-valo } \\
\hline \multirow{2}{*}{\multicolumn{2}{|c|}{ Categoria da CIF }} & \multicolumn{5}{|c|}{$\begin{array}{c}\text { Alguma } \\
\text { deficiência }\end{array}$} & \multicolumn{3}{|c|}{$\begin{array}{c}\text { Sem } \\
\text { deficiência }\end{array}$} & & & & & & & & & & & & \\
\hline & & 1 & 2 & 3 & 4 & $\%$ & 0 & 9 & $\%$ & 1 & 2 & 7 & 9 & $\%$ & 1 & 2 & 3 & $\%$ & & & \\
\hline s6302 & $\begin{array}{l}\text { Mamas e } \\
\text { mamilos }\end{array}$ & 0 & 23 & 7 & 0 & 100 & 0 & 0 & 0 & 7 & 23 & 0 & 0 & 100 & 12 & 18 & 0 & 100 & 2,23 & 2 & 0,003 \\
\hline s7201 & $\begin{array}{l}\text { Articulações } \\
\text { da região } \\
\text { do ombro }\end{array}$ & 17 & 11 & 1 & 0 & 97 & 1 & 0 & 3 & 0 & 0 & 30 & 0 & 100 & 12 & 18 & 0 & 100 & 1,40 & 1 & 0,000 \\
\hline s73001 & $\begin{array}{l}\text { Articulações } \\
\text { da região } \\
\text { do cotovelo }\end{array}$ & 26 & 0 & 2 & 0 & 93 & 2 & 0 & 7 & 0 & 0 & 30 & 0 & 100 & 12 & 18 & 0 & 100 & 1,06 & 1 & 0,000 \\
\hline s73011 & $\begin{array}{l}\text { Articulações } \\
\text { da região } \\
\text { do punho }\end{array}$ & 22 & 2 & 0 & 0 & 80 & 6 & 0 & 20 & 0 & 0 & 30 & 0 & 100 & 12 & 18 & 0 & 100 & 0,86 & 1 & 0,000 \\
\hline s8102 & $\begin{array}{l}\text { Pele do } \\
\text { membro } \\
\text { superior }\end{array}$ & 0 & 5 & 10 & 0 & 50 & 15 & 0 & 50 & 0 & 0 & 0 & 30 & 100 & 12 & 18 & 0 & 100 & 1,33 & 1 & 0,082 \\
\hline s840 & $\begin{array}{l}\text { Estrutura } \\
\text { dos pelos }\end{array}$ & 21 & 4 & 1 & 4 & 100 & 0 & 0 & 0 & 4 & 26 & 0 & 0 & 100 & 0 & 0 & 30 & 100 & 1,60 & 1 & 0,000 \\
\hline$s 420$ & $\begin{array}{l}\text { Estrutura } \\
\text { do sistema } \\
\text { imunológico }\end{array}$ & 0 & 30 & 0 & 0 & 100 & 0 & 0 & 0 & 0 & 0 & 0 & 30 & 100 & 12 & 18 & 0 & 100 & 2,00 & 2 & 0,000 \\
\hline
\end{tabular}

Legenda: CIF = Classificaçăo Internacional de Funcionalidade, Incapacidade e Saúde.

Nota: Qualificadores (extensão/ natureza/ localização): 1º Qualificador (0- nenhuma deficiência/ 1- deficiência leve/ 2- deficiência moderada/ 3- deficiência grave/ 4- deficiência completa/ 9- não aplicável); ${ }^{\circ}$ Qualificador (1- ausência total/ 2- ausência parcial/ 7- mudança qualitativa na estrutura, incluindo acúmulo de líquidos/ 9- não aplicável); 30 Qualificador (1- direita/ 2- esquerda/ 3- ambos os lados).

foi encontrado no estudo de Marinho e Macedo ${ }^{14}$, que relataram complicaçóes de movimentos do membro superior homolateral à cirurgia relacionadas ao tratamento radioterápico, à imobilização pós-cirurgia, à presença da síndrome do cordão axilar e a linfedema.

A função dor no membro superior (b28014) operado também foi um dado frequente, assim como a função tátil (b265). Bezerra et al. ${ }^{15}$ conduziram um estudo longitudinal acerca dessa temática, afirmando que o sistema sensorial em mulheres que passaram por cirurgia oncológica mamária pode ser comprometido, como a dor, que pode surgir logo no pós-operatório imediato e persistir com o tratamento radioterápico induzindo reaçóes cutâneas e nervosas, bem como o medo de mobilização e a própria inflamação decorrente da incisão cirúrgica. Além disso, o tratamento radioterápico em algumas mulheres induz a hipoestesia, juntamente com disfunçōes neurais como neuropatias decorrentes de fibrose e isquemia do tecido neural no local do tratamento, favorecendo a dor mesmo em pós-cirúrgico tardio ${ }^{16}$.

$\mathrm{O}$ acometimento doloroso pode comprometer ainda a estabilidade psíquica (b1263), o sono (b134), a confiabilidade (b1267) e o lidar com estresse e responsabilidade (d2400), categorias estas que apresentaram uma alta frequência de qualificadores com alteração grave ${ }^{15,16}$. Esses prejuízos afetam a capacidade e o desempenho de envolver-se em atividades do dia a dia, pois acarretam situaçóes de sofrimento, ansiedade, angústia e baixa autoestima ${ }^{6}$. Desta forma, é fundamental que a mulher receba assistência com uma equipe multiprofissional sob abordagem interdisciplinar que seja capaz de amenizar os sofrimentos em diversos aspectos e contextos $^{17}$.

Tarefas como executar tarefas múltiplas (d2200), tarefas domésticas (d640), concluir rotina diária (d2302), transportar nas mãos (d4301), empurrar (d4451) e 
Tabela 3. Frequência das categorias de função corporal da CIF, de acordo com os qualificadores

\begin{tabular}{|c|c|c|c|c|c|c|c|c|c|c|c|c|}
\hline \multicolumn{13}{|c|}{ b- Função corporal } \\
\hline & \multirow{3}{*}{ Categoria da CIF } & \multicolumn{8}{|c|}{ Qualificador da CIF } & \multirow{3}{*}{ Média } & \multirow{3}{*}{ Mediana } & \multirow{3}{*}{ p-valor } \\
\hline & & \multicolumn{5}{|c|}{ Alguma deficiência } & \multicolumn{3}{|c|}{ Sem deficiência } & & & \\
\hline & & 1 & 2 & 3 & 4 & $\%$ & 0 & 9 & $\%$ & & & \\
\hline bl1420 & Orientação em relação a si próprio & 10 & 0 & 2 & 3 & 50,0 & 15 & 0 & 50,0 & 0,93 & 0,5 & 0,002 \\
\hline b1263 & Estabilidade psíquica & 9 & 0 & 0 & 7 & 53,3 & 14 & 0 & 46,7 & 1,23 & 1 & 0,273 \\
\hline b1267 & Confiabilidade & 2 & 0 & 11 & 5 & 60,0 & 12 & 0 & 40,0 & 1,83 & 3 & 0,027 \\
\hline bl300 & Nível de energia & 12 & 0 & 0 & 11 & 76,7 & 7 & 0 & 23,3 & 1,86 & 1 & 0,497 \\
\hline b134 & Funções do sono & 3 & 2 & 9 & 3 & 56,7 & 13 & 0 & 43,3 & 1,53 & 1 & 0,004 \\
\hline bl344 & Funções que envolvem o ciclo do sono & 7 & 0 & 0 & 12 & 63,3 & 11 & 0 & 36,7 & 1,83 & 1 & 0,497 \\
\hline bl400 & Manutenção da atenção & 6 & 0 & 0 & 7 & 43,3 & 17 & 0 & 56,7 & 1,13 & 0 & 0,025 \\
\hline b1441 & Memória de longo prazo & 7 & 0 & 0 & 13 & 66,7 & 10 & 0 & 33,3 & 1,96 & 1 & 0,407 \\
\hline bl800 & Experiencia pessoal & 6 & 0 & 0 & 8 & 46,7 & 16 & 0 & 53,3 & 1,26 & 0 & 0,061 \\
\hline bl801 & Imagem do corpo & 5 & 0 & 0 & 4 & 30,0 & 21 & 0 & 70,0 & 0,70 & 0 & 0,000 \\
\hline b250 & Função gustativa & 7 & 0 & 0 & 7 & 46,7 & 16 & 0 & 53,3 & 1,16 & 0 & 0,067 \\
\hline b265 & Função tátil & 8 & 0 & 0 & 12 & 66,7 & 10 & 0 & 33,3 & 1,86 & 1 & 0,670 \\
\hline b28010 & Dor na cabeça ou pescoço & 11 & 0 & 0 & 5 & 53,3 & 13 & 1 & 46,7 & 1,33 & 1 & 0,007 \\
\hline b28011 & Dor no peito & 12 & 0 & 0 & 9 & 70,0 & 9 & 0 & 30,0 & 1,60 & 1 & 0,741 \\
\hline b28014 & Dor em membro superior & 3 & 8 & 13 & 1 & 83,3 & 5 & 0 & 16,7 & 2,07 & 2 & 0,005 \\
\hline b28018 & Dor localizada & 7 & 0 & 0 & 8 & 50,0 & 14 & 1 & 50,0 & 1,60 & 1 & 0,010 \\
\hline b440 & Funções da respiração & 8 & 0 & 0 & 4 & 40,0 & 18 & 0 & 60,0 & 0,80 & 0 & 0,006 \\
\hline b5104 & Salivação & 5 & 0 & 0 & 13 & 60,0 & 11 & 1 & 40,0 & 2,20 & 1 & 0,007 \\
\hline b5106 & Regurgitação e vômito & 3 & 0 & 0 & 2 & 16,7 & 24 & 1 & 83,3 & 0,66 & 0 & 0,000 \\
\hline b5250 & Eliminação de fezes & 9 & 0 & 0 & 7 & 53,3 & 14 & 0 & 46,7 & 1,23 & 1 & 0,273 \\
\hline b5253 & Continência fecal & 4 & 0 & 0 & 1 & 16,7 & 25 & 0 & 83,3 & 0,26 & 0 & 0,000 \\
\hline b5350 & Sensação de náusea & 6 & 0 & 0 & 4 & 33,3 & 20 & 0 & 66,7 & 0,73 & 0 & 0,001 \\
\hline b6400 & Funções da fase de excitação sexual & 11 & 0 & 0 & 9 & 66,7 & 10 & 0 & 33,3 & 1,56 & 1 & 0,905 \\
\hline b6402 & Funções da fase orgásmica & 9 & 0 & 0 & 7 & 53,3 & 2 & 12 & 46,7 & 8,50 & 4 & 0,000 \\
\hline b7100 & $\begin{array}{l}\text { Mobilidade de uma única articulação } \\
\text { (abdução de ombro) }\end{array}$ & 17 & 11 & 2 & 0 & 100,0 & 0 & 0 & 0,0 & 1,50 & 1 & 0,003 \\
\hline b7100 & $\begin{array}{l}\text { Mobilidade de uma única articulação } \\
\text { (flexão de ombro) }\end{array}$ & 16 & 8 & 4 & 0 & 93,3 & 2 & 0 & 6,7 & 1,46 & 1 & 0,002 \\
\hline b7100 & $\begin{array}{l}\text { Mobilidade de uma única articulação } \\
\text { (extensão de ombro) }\end{array}$ & 13 & 3 & 3 & 0 & 63,3 & 11 & 0 & 36,7 & 0,93 & 1 & 0,011 \\
\hline b7100 & $\begin{array}{l}\text { Mobilidade de uma única articulação } \\
\text { (flexão de cotovelo) }\end{array}$ & 4 & 0 & 0 & 0 & 13,3 & 26 & 0 & 86,7 & 0,13 & 0 & 0,000 \\
\hline b7200 & Mobilidade de ombro & 4 & 5 & 2 & 7 & 60,0 & 12 & 0 & 40,0 & 1,60 & 1 & 0,046 \\
\hline b7300 & $\begin{array}{l}\text { Força de músculos isolados e grupos } \\
\text { musculares }\end{array}$ & 7 & 0 & 0 & 18 & 83,3 & 5 & 0 & 16,7 & 2,63 & 4 & 0,007 \\
\hline b740 & Funções da resistência muscular & 3 & 0 & 0 & 15 & 60,0 & 12 & 0 & 40,0 & 2,10 & 2,5 & 0,020 \\
\hline b7800 & Sensação de rigidez muscular & 3 & 6 & 5 & 2 & 53,3 & 14 & 0 & 46,7 & 1,26 & 1 & 0,005 \\
\hline
\end{tabular}

Legenda: CIF = Classificação Internacional de Funcionalidade, Incapacidade e Saúde.

Nota: Qualificador (0- nenhuma deficiência/ 1- deficiência leve/ 2- deficiência moderada/ 3- deficiência grave/ 4- deficiência completa/ 9- não aplicável). 
Tabela 4. Frequência das categorias dos domínios atividade e participação da ClF, de acordo com os qualificadores de 30 voluntárias submetidas à cirurgia oncológica mamária

\begin{tabular}{|c|c|c|c|c|c|c|c|c|c|c|c|c|}
\hline \multicolumn{13}{|c|}{ d-Atividade e participação (desempenho) } \\
\hline & \multirow{3}{*}{ Categoria da CIF } & \multicolumn{8}{|c|}{ Qualificador da CIF } & \multirow{3}{*}{ Média } & \multirow{3}{*}{ Mediana } & \multirow{3}{*}{ p-valor } \\
\hline & & \multicolumn{5}{|c|}{ Alguma restrição } & \multicolumn{3}{|c|}{ Sem restrição } & & & \\
\hline & & 1 & 2 & 3 & 4 & $\%$ & 0 & 9 & $\%$ & & & \\
\hline $\mathrm{d} 170$ & Escrever & 3 & 0 & 2 & 3 & 26,7 & 22 & 0 & 73,3 & 0,70 & 0 & 0,000 \\
\hline $\mathrm{d} 2200$ & Executar tarefas múltiplas & 9 & 0 & 9 & 1 & 66,3 & 11 & 0 & 36,7 & 1,33 & 1 & 0,049 \\
\hline d2302 & Concluir a rotina diária & 8 & 0 & 0 & 14 & 73,3 & 7 & 1 & 26,6 & 2,43 & 2,5 & 0,010 \\
\hline d2303 & Gerir o seu próprio nível de atividade & 13 & 0 & 4 & 3 & 66,6 & 10 & 0 & 33,3 & 1,23 & 1 & 0,027 \\
\hline $\mathrm{d} 2400$ & Lidar com responsabilidades & 10 & 0 & 0 & 12 & 73,3 & 8 & 0 & 26,7 & 1,93 & 1 & 0,670 \\
\hline d2401 & Lidar com estresse & 5 & 0 & 0 & 13 & 60 & 12 & 0 & 40,0 & 1,90 & 1 & 0,150 \\
\hline d4104 & Pôr-se em pé & 8 & 0 & 0 & 6 & 46,7 & 16 & 0 & 53,3 & 1,06 & 0 & 0,061 \\
\hline d4301 & Transportar nas mãos & 7 & 4 & 5 & 7 & 76,6 & 7 & 0 & 23,3 & 1,93 & 2 & 0,856 \\
\hline d4451 & Empurrar & 6 & 4 & 2 & 5 & 56,7 & 13 & 0 & 43,3 & 1,33 & 1 & 0,020 \\
\hline d4452 & Alcançar & 5 & 4 & 4 & 8 & 70 & 9 & 0 & 30,0 & 1,90 & 2 & 0,453 \\
\hline$d 4453$ & Rodar ou torcer as mãos ou os braços & 7 & 5 & 3 & 9 & 80 & 6 & 0 & 20,0 & 2,07 & 2 & 0,504 \\
\hline d5100 & Lavar partes do corpo & 9 & 3 & 3 & 7 & 73,3 & 8 & 0 & 26,7 & 1,73 & 1 & 0,255 \\
\hline d5101 & Lavar todo o corpo & 9 & 0 & 0 & 4 & 43,3 & 17 & 0 & 56,7 & 0,83 & 0 & 0,014 \\
\hline$d 5202$ & Cuidar do cabelo e da barba & 4 & 2 & 4 & 3 & 43,3 & 17 & 0 & 56,7 & 1,06 & 0 & 0,000 \\
\hline$d 5400$ & Vestir roupa & 6 & 3 & 3 & 6 & 60 & 12 & 0 & 40,0 & 1,50 & 1 & 0,061 \\
\hline $\mathrm{d} 550$ & Comer & 1 & 5 & 4 & 5 & 50 & 15 & 0 & 50,0 & 1,43 & 0,5 & 0,001 \\
\hline$d 5700$ & Assegurar o próprio conforto físico & 9 & 0 & 1 & 0 & 33,3 & 20 & 0 & 66,7 & 0,40 & 0 & 0,000 \\
\hline d5702 & Manter a própria saúde & 8 & 4 & 5 & 2 & 63,4 & 11 & 0 & 36,7 & 1,30 & 1 & 0,080 \\
\hline$d 6300$ & Preparar refeições simples & 2 & 5 & 6 & 3 & 53,4 & 14 & 0 & 46,7 & 1,40 & 1 & 0,005 \\
\hline $\mathrm{d} 640$ & Realizar tarefas domésticas & 5 & 1 & 5 & 11 & 73,3 & 8 & 0 & 26,7 & 2,20 & 3 & 0,053 \\
\hline $\mathrm{d} 6501$ & Manutenção da habitação e dos móveis & 2 & 0 & 2 & 14 & 60 & 12 & 0 & 40,0 & 2,13 & 3 & 0,001 \\
\hline$d 6505$ & Cuidar das plantas do interior e exterior & 5 & 4 & 1 & 8 & 60 & 12 & 0 & 40,0 & 1,60 & 1 & 0,020 \\
\hline$d 7108$ & Interações interpessoais básicas & 10 & 0 & 0 & 7 & 56,6 & 13 & 0 & 43,3 & 1,26 & 1 & 0,407 \\
\hline $\mathrm{d} 7608$ & Relacionamentos familiares & 7 & 0 & 0 & 7 & 46,6 & 16 & 0 & 53,3 & 1,16 & 0 & 0,067 \\
\hline $\mathrm{d} 7702$ & Relacionamentos sexuais & 0 & 6 & 1 & 6 & 43,3 & 17 & 0 & 56,7 & 1,30 & 0 & 0,000 \\
\hline d8451 & Manter um emprego & 7 & 0 & 0 & 10 & 56,6 & 13 & 0 & 43,3 & 1,56 & 1 & 0,407 \\
\hline$d 8508$ & Trabalho remunerado & 7 & 0 & 0 & 10 & 56,6 & 13 & 0 & 43,3 & 1,56 & 1 & 0,407 \\
\hline $\mathrm{d} 8700$ & Recursos econômicos pessoais & 3 & 0 & 0 & 9 & 40 & 18 & 0 & 60,0 & 1,30 & 0 & 0,003 \\
\hline d9205 & Socialização & 4 & 0 & 0 & 3 & 23,3 & 23 & 0 & 76,7 & 0,53 & 0 & 0,000 \\
\hline
\end{tabular}

Legenda: $\mathrm{CIF}=$ Classificaçáo Internacional de Funcionalidade, Incapacidade e Saúde.

Nota: Qualificador (0- nenhuma restriçăo/ 1- restriçăo leve/ 2- restriçāoo moderada/ 3- restriçăo grave/ 4- restriçăo completa/ 9- nâo aplicável).

lavar as partes do corpo (d5100) envolvem açôes de movimentos repetitivos e em várias direçóes dos MMSS. No presente estudo, essas tarefas foram frequentemente qualificadas com maior gravidade, o que é justificado pelas alteraçóes dos movimentos de abdução e flexão do ombro (b7100) e força de músculos isolados (b7300) encontrados nessas mulheres ${ }^{18}$. Essa interdependência das categorias mostra o quanto é preciso conhecer o contexto de vida da mulher, isto é, se é casada, se cuida da casa, se trabalha fora, se é responsável pela família, como também identificar as limitações de atividades que envolvem o seu dia a dia e investir no tratamento fisioterapêutico com ações centradas no indivíduo, para a recuperação dos movimentos que são essenciais para a sua independência e autonomia.

Se por um lado passar por uma cirurgia oncológica mamária esteja relacionado com uma experiência negativa, a CIF nos propóe um olhar mais ampliado, em que é 
viável vislumbrar elementos que possam fortalecer o processo de tratamento e recuperação, aumentando a funcionalidade ${ }^{19}$. Neste estudo, grande parte das mulheres submetidas à cirurgia oncológica mamária apresentou qualificadores sem restrição como o autocuidado (d5700; d5101; d5202), recursos econômicos (d8700), relação familiar (d7608) e social. Essas categorias refletem que, mesmo a mulher passando por um momento delicado, elas asseguraram o próprio cuidado, tinham uma boa relação familiar e bons recursos econômicos, otimizaram o acesso aos serviços de saúde, favorecendo a funcionalidade ${ }^{13}$.

A CIF possui um core set específico para o câncer de mama, com categorias elencadas para sua qualificação. Entretanto, ao se compararem as categorias selecionadas neste estudo com o core set, verificou-se que os componentes "atividade e participação" apresentaram maior número de itens. Carvalho et al. ${ }^{20}$ realizaram um estudo similar e identificaram categorias referentes à "atividade e participaçáo" não contempladas pelo core set. Esses autores justificaram que o core set se baseia na escolha das categorias na doença, limitando o olhar no que de fato importa identificar o que o indivíduo é capaz ou não de realizar no seu dia a dia ${ }^{20}$.

Por fim, é interessante destacar que a ficha de avaliação fisioterapêutica estruturada para o serviço de oncologia do local de realização desta pesquisa contempla todos os componentes da $\mathrm{CIF}^{21}$. Entretanto, observa-se que existe uma alta representatividade do componente "função do corpo" no modelo de avaliação e, ao mesmo tempo, poucas categorias elencadas em "fatores ambientais", o que condiz com uma limitaçáo do estudo. Contudo, o componente "atividade e participaçáa" foi bem representado, sugerindo alta influência na determinação da funcionalidade e incapacidade de mulheres com câncer de mama.

\section{CONCLUSÃO}

Mulheres submetidas à cirurgia oncológica mamária apresentam alteraçóes na estrutura do corpo, como mama e mamilos, articulação do ombro, pelos/cabelos; e função do corpo, no que tange à dor em membro superior e à funçáo articular nos movimentos de ombro, que limitam realizar atividades cotidianas, impactando negativamente na sua independência e autonomia. $\mathrm{O}$ uso de uma ficha de avaliaçáo que aborde todos os componentes da CIF proporciona a visão do indivíduo como um todo.

\section{CONTRIBUIÇÕES}

Os autores contribuíram de forma equivalente em todas as etapas do trabalho e aprovaram a versão a ser publicada.

\section{DECLARAÇÃO DE CONFLITO DE INTERESSES}

Nada a declarar.

\section{FONTES DE FINANCIAMENTO}

Coordenação de Aperfeiçoamento de Pessoal de Nível Superior (Capes). Código de financiamento 001.

\section{REFERÊNCIAS}

1. Organização Mundial da Saúde. Classificação Internacional de Funcionalidade, Incapacidade e Saúde. Sáo Paulo: EdUSP; 2008.

2. Stucki G, Reinhardt JD, Grimby G, et al. Developing "Human functioning and rehabilitation research" from the comprehensive perspective. J Rehabil Med. 2007;39(9):655-71. doi: https://doi. org/10.2340/16501977-0136

3. Harty M, Griesel M, van der Merwe A. The ICF as a common language for rehabilitation goal-setting: comparing client and professional priorities. Health Qual Life Outcomes. 2011;9:87. doi: https://doi. org/10.1186/1477-7525-9-87

4. Lourenço A, Dantas AAG, Araújo DN, et al. Prevalência da deficiência e associaçôes clínicas em mulheres sobreviventes ao câncer de mama: um estudo-piloto. Rev Bras de Cancerol. 2020;66(2): e-13843. doi: https://doi. org/10.32635/2176-9745.RBC.2020v66n2.843

5. Santos VA, Seippel T, Castaneda L. Utilização da classificaçáo internacional de funcionalidade, incapacidade e saúde em mulheres com câncer de mama: revisão da literatura. Rev Bras Cancerol. 2019;65(1): e-15349. doi: https://doi.org/10.32635/2176-9745. RBC.2019v65n1.349

6. Costa ID, Santos DHO, Silva VM, et al. Utilização de um core set da CIF para a descriçáo da atividade e participação de mulheres submetidas ao tratamento cirúrgico para o câncer de mama. Rev Interdiscip Ciênc Méd. 2018;1(2):4-14.

7. Castro SS, Castaneda L, Araújo ES, et al. Aferição de funcionalidade em inquéritos de saúde no Brasil: discussão sobre instrumentos baseados na Classificaçáo Internacional de Funcionalidade, Incapacidade e Saúde (CIF). Rev Bras Epidemiol. 2016;19(3):679-87. doi: https:// doi.org/10.1590/1980-5497201600030018

8. Drumond AS. Exploração do Disabilities Arm, Shoulder and Hand (DASH) através da Classificação Internacional de Funcionalidade, Incapacidade E Saúde (CIF) e da análise Rasch [dissertação]. Belo Horizonte: Universidade Federal de Minas Gerais; 2006.

9. Skrzypulec V, Tobor E, Drosdzol A, et al. Biopsychosocial functioning of women after mastectomy. J Clin Nurs. 
2009;18(4):613-9. doi: https://doi.org/10.1111/j.13652702.2008.02476.x

10. Fernandes MMJ, Alves PC, Santos MCL, et al. Autoestima de mulheres mastectomizadas - aplicaçáo da escala de Rosenberg. Rev Rene. 2013;14(1):101-8.

11. Baraúna MA, Canto RST, Schulz E, et al. Avaliação da amplitude de movimento do ombro em mulheres mastectomizadas pela biofotogrametria computadorizada. Rev Bras Cancerol. 2004;50(1):27-31. doi: https://doi. org/10.32635/2176-9745.RBC.2004v50n1.2053

12. Cieza A, Fayed N, Bickenbach J, et al. Refinements of the ICF Linking Rules to strengthen their potential for establishing comparability of health information. Disabil Rehabil. 2019;41(5):574-83. doi: https://doi.org/10.31 09/09638288.2016.1145258

13. Roberti BFA, Scudeller TT, Amaral MTP. Influência do tratamento do câncer de mama na funcionalidade do membro superior e no retorno à atividade laboral. Rev Ciênc Méd. 2016;25(2):69-76. doi: https://doi. org/10.24220/2318-0897v25n2a3564

14. Marinho ACN, Macedo AA. Análise da amplitude de movimento do ombro de mulheres mastectomizadas submetidas a um programa de exercícios e alongamentos musculares. Fisioter Bras. 2006;7(1):30-5. doi: https:// doi.org/10.33233/fb.v7i1.1861

15. Bezerra TS, Rett MT, Mendonça ACR, et al. Hipoestesia, dor e incapacidade no membro superior após radioterapia adjuvante no tratamento para câncer de mama. Rev Dor. 2012;13(4):320-326. doi: https://doi.org/10.1590/ S1806-00132012000400003

16. Ornelas FA, Rodrigues JR, Uemura G. Avaliação convencional e estesiômetro: resultados controversos na avaliação sensitiva no câncer de mama. HU Rev. 2010;36(2):137-145.

17. Rozario GF, Rodrigues TCR, Galaverna LS, et al. Aplicação da Escala de Rosenberg para Avaliação da Autoestima de Mulheres Submetidas à Cirurgia Oncológica Mamária. Rev Saúde Foco. 2019;6(2):66-85. doi: http://doi.org/10.12819/rsf.2019.6.2.6

18. Bruce J, Thornton AJ, Powell R, et al. Psychological, surgical, and sociodemographic predictors of pain outcomes after breast cancer surgery: a population-based cohort study. Pain. 2014;155(2):232-43. doi: http://doi. org/10.1016/j.pain.2013.09.028

19. Correia T, Monteiro S, Torres A, et al. Eficácia da intervenção multidisciplinar em sobreviventes de cancro de mama. Psicol Saúde Doenças. 2016;17(3):483-502. doi: http://doi.org/10.15309/16psd170314

20. Carvalho FN, Koifman RJ, Bergmann A. International Classification of Functioning, Disability, and Health in women with breast cancer: a proposal for measurement instruments. Cad Saúde Pública. 2013;29(6):1083-93. doi: https://doi.org/10.1590/ S0102-311X2013000600005

21. Araujo ES, Buchalla CM. O uso da Classificação Internacional de Funcionalidade, Incapacidade e Saúde em inquéritos de saúde: uma reflexão sobre limites e possibilidades. Rev Bras Epidemiol. 2015;18(3):720-24. doi: https://doi.org/10.1590/1980-5497201500030017

Recebido em 28/1/2021 Aprovado em 19/3/2021 